\title{
El análisis didáctico como base de un curso en la formación inicial de profesores de educación primaria
}

\author{
Macarena Valenzuela-Molina, Elisabeth Ramos-Rodríguez, Lylian-Iris González-Plate y \\ Jocelyn-Lysette Portugal-Villar
}

\section{RESUMEN}

El diseño e implementación de un curso de formación inicial para profesores de matemáticas que ayude a comprender cómo los estudiantes se relacionan con el conocimiento profesional suministrado en ellos, ha sido nuestro foco. Partimos de la concepción de proceso formativo que aborda una visión funcional del conocimiento didáctico del contenido, encaminado a profundizar sobre el significado de las tareas matemáticas que están involucradas en las planificaciones de clases diseñadas por futuros profesores de tres universidades chilenas. Desde el paradigma cualitativo, esta investigación tiene por objetivo mostrar de qué manera les es útil a los futuros profesores la herramienta análisis didáctico, como procedimiento para diseñar, implementar y evaluar las clases. Comprender estos elementos nos permite diseñar cursos más apropiados a la realidad de la formación inicial en las carreras de pedagogía de universidades chilenas.

Palabras clave: análisis didáctico, matemática, formación de profesores, Chile.

Macarena Valenzuela-Molina mvalenzuelamolina@gmail.com Chilena. Doctoranda, Pontificia Universidad Católica de Valparaíso, Chile. Profesora, Universidad Alberto Hurtado, Chile. Temas de investigación: formación de profesores, desarrollo profesional docente, didáctica de la aritmética.

Elisabeth Ramos-Rodríguez

elisabeth.ramos@pucv.cl Chilena. Doctora en Ciencias de la Educación, Universidad de Granada. Profesora asociada, Pontificia Universidad Católica de Valparaíso, Chile. Temas de investigación: formación de profesores, desarrollo profesional docente, didáctica de la matemática, modelación matemática.

Lylian-Iris González-Plate

Iylian.gonzalez@pucv.cl

Chilena. Doctora, Universidad de Granada, España. Profesora Jerarquía Adjunto, Pontificia Universidad Católica de Valparaíso, Chile. Temas de investigación: formación inicial de docentes, educación física escolar, didáctica de la educación física, concepciones en torno a la corporeidad. saber pedagógico, identidad profesional. 


\title{
A análise didática como base de um curso na formação inicial de professores de educação primária
}

\section{RESUMO}

A Planificação e implementação de um curso de formação inicial para professores de matemática que ajude a compreender como os estudantes se relacionam com o conhecimento profissional subministrado neles, tem sido nosso foco. Partimos da concepção de processo formativo que aborda uma visão funcional do conhecimento didático de conteúdo, encaminhado a aprofundar sobre o significado das tarefas matemáticas que estão envolvidas nas planificações de aulas feitas por futuros professores de três universidades chilenas. Desde o paradigma qualitativo, esta pesquisa tem por objetivo mostrar de que maneira lhes será útil aos futuros professores, a ferramenta análisis didáctico, como procedimento para planificar, implementar e avaliar as aulas. Compreender estes elementos nos permite planejar cursos mais apropriados a realidade da formação inicial nas carreiras de pedagogia de universidades chilenas.

Palavras chave: análise didática, matemática, formação de professores, Chile.

\section{The didactic analysis as a basis for a course in the initial training of primary school teachers}

\begin{abstract}
The design and implementation of an initial training course for teachers of mathematics to help understand how students relate to the professional knowledge provided in them, has been our focus. The authors start from the conception of formative process that approaches a functional view of the didactic knowledge of content, aimed at deepening on the meaning of the mathematical tasks that are involved in the lesson plans designed by future professors of three Chilean universities. From the qualitative paradigm, this research aims to show how the didactic analysis tool is useful to future teachers as a procedure to design, implement and evaluate the classes. Understanding these elements allows us to design courses more appropriate to the reality of initial training in the degrees of Chilean universities.
\end{abstract}

Key words: didactic analysis, mathematics, teacher training, Chile.

Recepción: 24/02/16. Aprobación: 09/05/17. 


\section{Introducción y antecedentes}

Chile participó en el estudio comparativo TEDS-M ${ }^{1}$ del año 2008 para profesores en formación de educación primaria de diversos países. Nuestro país obtuvo puntajes bajo la media, evidenciando deficiencias en contenido disciplinar y pedagógico, lo que pone en discusión la preparación de la formación inicial (Blömeke, Suhl y Kaiser, 2011).

Frente a esto se cuenta con el Programa de Fomento a la Calidad de la Formación Inicial Docente (Ministerio de Educación [MINEDUC], 2012) dentro del cual se encuentra la Evaluación INICIA que evalúa las competencias de los egresados de pedagogía, inmediatamente después de su egreso. Los resultados de la prueba INICIA de 2013 arrojan deficiencias, con una baja mayor en lo disciplinario respecto a la pedagógico, poniendo bajo cuestionamiento el conocimiento que deben tener los profesores recién egresados.

Investigadores como Ávalos (2002), Cox (2007), Ortúzar, Flores, Milesi y Cox (2009), Pedraja-Rejas, Araneda-Guirriman, Rodríguez-Ponce y RodríguezPonce (2012), coinciden respecto a la necesidad de mejorar y replantear la formación inicial docente chilena, siendo uno de los focos de atención el conocimiento que debe tener un profesor para desempeñar su labor. El futuro profesor debe ser capaz de coordinar su formación didáctica con el conocimiento específico de una disciplina en particular, los profesores son los sujetos que unen la teoría con la acción práctica (Villar, 1986).

Desde una perspectiva teórica, algunos investigadores de la didáctica de las matemáticas han propuesto una herramienta para la enseñanza y el aprendizaje de esta área, el análisis didáctico, basada en el concepto de currículo, cuyo objetivo es proporcionar a los estudiantes de pedagogía, un procedimiento de planificación que les permita diseñar una unidad didáctica, fundamentarla, justificar su diseño y llevarla a la práctica (Gómez, 2007).

Este procedimiento de planificación se llevó a cabo con un grupo de estudiantes de la carrera de Educación Básica, ${ }^{2}$ con mención en matemática, de la Universidad Alberto Hurtado, durante dos semestres completos, en el marco del proyecto "Aprendizaje docente y desarrollo profesional en experiencias de enseñanza de la Educación Física y la Matemática en el Primer ciclo de Enseñanza Básica: Una mirada desde las representaciones de los estudiantes de Pedagogía en Educación Básica", que incluye a dos universidades chilenas más, la Pontificia Universidad Católica de Valparaíso $^{3}$ y la Universidad Santo Tomás.

El desarrollo de los trabajos de los estudiantes fue complejo. Debido a que no tenían conocimientos sobre esta herramienta, tuvieron que ir profundizando en ella a medida que diseñaban su trabajo final de titulación. Sin embargo, los resultados obtenidos por ellos fueron muy satisfactorios, ya que lograron realizar exitosamente todo el procedimiento implicado en el análisis didáctico, profundizando en conceptos matemáticos que deberán manejar en su práctica pedagógica, siendo capaces de diseñar e implementar una unidad didáctica consistente en 16 horas pedagógicas, en distintos colegios de Santiago, en donde realizaban sus primeras experiencias laborales. La reflexión personal y la reflexión acompañada por el tutor didacta, les permitieron analizar en profundidad la implementación de su unidad.

En el desarrollo de unidades didácticas, hemos constatado que las tareas escolares diseñadas por los estudiantes de la carrera de Educación Primaria son un potente contexto de estudio. En ellas se evidencian los conocimientos matemáticos y didácticos que poseen nuestros futuros profesores, conocimientos que pondrán en juego cuando les corresponda entrar al campo laboral.

\footnotetext{
${ }^{1}$ Estudio internacional sobre formación inicial en matemáticas de maestros.

${ }^{2}$ En Chile la educación básica comprende los años escolares para los niños de 6 a 13 años aproximadamente.

${ }^{3}$ Agradecemos a la Pontificia Universidad Católica de Valparaíso por ser la institución financiadora de este estudio.
} 
El propósito de nuestro estudio es mostrar de qué manera les es útil a futuros profesores, la herramienta análisis didáctico $(\mathrm{AD})$, como procedimiento para diseñar, implementar y evaluar las clases.

$\mathrm{El} \mathrm{AD}$ en la formación inicial de profesores se ha utilizado esmeradamente en proyectos de innovación y divulgado en diferentes publicaciones del grupo Pensamiento Numérico Algebráico (PNA) de la Universidad de Granada (Gómez y González, 2013; Lupiáñez, y Rico, 2006; 2008). También se manifiesta el uso y aplicación del AD en libros de texto de Segovia y Rico (2011) y de Flores y Rico (2015) y se ha publicado el libro "Análisis didáctico en educación matemática" (Rico, Lupiáñez y Molina, 2013), que reúne una gran cantidad de trabajos e investigaciones sobre el AD como metodología de investigación, metodología de formación de profesores e innovación curricular. También se ha concretado en diversos trabajos de fin de maestrías en universidades españolas (León, 201 1; Ortega, 2012). Nuestro equipo también ha liderado trabajos de fin de carrera, tanto en la formación inicial de pedagogía en educación primaria (Ortiz, 2013; Ruiz, 2013) como en educación media (Aros y Astudillo, 2015; Fonseca y Reyes, 2015).

Como ya hemos mencionado, esta herramienta se viene utilizando desde hace años, en el departamento de Didáctica de la Matemática de la Universidad de Granada (UGR), para la formación inicial de profesores de matemáticas de Enseñanza Secundaria (Rico, 1997a; 1997b), y ha dado lugar a tesis de doctorado (Gómez, 2007; Lupiáñez, 2009; Rojas, 2014). Por lo tanto, nuestro interés se centra en observar de qué manera les es útil el $\mathrm{AD}$ a los futuros profesores chilenos, como procedimiento para diseñar, implementar y evaluar las clases.

\section{Marco de referencia}

El sustento teórico de este estudio se basa en la noción de tarea y de análisis didáctico, elementos que detallaremos a continuación.

Para estudiar la tarea, será necesario explicitar los elementos que las definen (Doyle, 1933; Ponte, 2005): la meta u objetivo de aprendizaje, la forma en que se formula u organiza (enunciado), el contenido disciplinario, el tipo de materiales y formas de comunicarse; estas dimensiones objetivas nos facilitan describir cómo son las tareas. Si queremos avanzar, debemos buscar nuevas dimensiones de análisis, con un carácter más valorativo. Estudiar la formulación y la coherencia entre la tarea propuesta y la meta que se pretende alcanzar, parecen ser dos dimensiones importantes (Flores, Gómez y Marín, 2013), por lo que tendremos que aclarar cómo podemos examinar esta coherencia.

Entenderemos que una tarea es coherente si tras el análisis de ésta se pueden describir los elementos que están presentes en ella, que corresponden con los seleccionados para la unidad didáctica. Hablaremos de que una tarea tiene baja, mediana o alta coherencia dependiendo del grado en que los elementos anteriores se hacen presentes.

$\mathrm{El}$ análisis didáctico es una herramienta para el profesor o futuro profesor, basado en la teoría de currículo, ${ }^{4}$ que le permite diseñar, poner en práctica y evaluar unidades didácticas en la enseñanza de las matemáticas (Lupiáñez, 2009). Esta herramienta de planificación, propuesta por Rico (1997b), responde a cuatro cuestiones esenciales: ¿Qué es el conocimiento matemático? ¿Qué es el aprendizaje? ¿Qué es la enseñanza? ¿Para qué sirve el conocimiento? Estas interrogantes se relacionan con cuatro dimensiones del currículo: cultural y conceptual; cognitiva o de desarrollo; ética o formativa, y la dimensión social, que permiten organizar y relacionar la legislación educativa en la planificación docente. Cada una de estas dimensiones se puede concretar en diferentes niveles, que dependen de las personas o

\footnotetext{
${ }^{4}$ Rico (1997b) nos habla del concepto de currículo identificando toda actividad de formación en la educación obligatoria del currículo.
} 
instituciones involucradas: planificación del docente, sistema educativo, disciplinas académicas y teológicas (Rico, 1997a).

Según Ruiz (2009) es necesario que los docentes reflexionen sobre su propia práctica para llevar a cabo una planificación y una reflexión que se sustente en una fundamentación disciplinar objetiva, donde la reflexión es parte fundamental en la concreción del currículo en el aula. Para ello, Rico (2007) introduce la noción de organizadores del currículo de matemáticas, definiéndolos como "aquellos conocimientos que adoptamos como componentes fundamentales para articular el diseño, desarrollo y evaluación de unidades didácticas". Entre los organizadores se encuentran (Rico, 1997b; Rico y Segovia, 2001): la estructura de los contenidos; el análisis fenomenológico de los conocimientos matemáticos; las representaciones; los modelos matemáticos y los procesos de modelización; los errores, dificultades y obstáculos asociados a conceptos y procedimientos de cada tema matemático; los materiales y recursos; la historia de las matemáticas, o la dimensión cultural e histórica del conocimiento matemático, y los estudios sobre resolución de problemas.

A partir de estos organizadores del currículo, se constituye el análisis didáctico, tal como lo concibe el grupo PNR, de la UGR, en España (Gómez, 2007, Lupiáñez y Rico, 2008; Rico, 1997a, 1997b; Rico, Lupiáñez y Molina, 2013), y consiste en una estrategia de análisis que se subdivide en cinco subanálisis (figura 1): conceptual, contenido, cognitivo, instrucción y actuación (Rico et al., 2013). Este análisis permite profundizar y elaborar tareas apropiadas de acuerdo con el tema que corresponde trabajar en la sala de clases. Se espera que este procedimiento sea realizado de manera ideal por los futuros profesores, describiendo todos los procedimientos sistemáticos y secuenciados.

El primer análisis es el conceptual, permite entender e interpretar los procesos mentales y psicológicos de determinados conceptos, es un método que nos permite convertir los conceptos en piezas teóricas precisas, examinando la diversidad de significados, las posibilidades de conexión entre los términos y los niveles subjetivos (creencias), intersubjetivos (concepciones) y objetivos (conceptos) de cada campo conceptual.

El análisis de contenido, es una técnica reductora, que permite descomponer el contenido en sus unidades más simples, en matemática permite estudiar la diversidad de significados escolares de los conceptos y sus procedimientos (Rico y Fernández-Cano, 2013). En él se estudia la estructura y análisis formal que abarca los conceptos, definiciones y procedimientos, sistemas de representación, que comprende las diferentes notaciones concretas, gráficas simbólicas y sistemas de signos involucrados; y el análisis fenomenológico, que trata los fenómenos, contextos y situaciones que dan origen y en los cuales se pueden dar sentido a los contenidos matemáticos en estudio (Rico y Fernández-Cano, 2013).

El análisis cognitivo, organiza el "para qué" y "hasta dónde" aprender ciertos conocimientos para un tema en específico. Se compone de tres categorías, la primera de ellas son las expectativas de aprendizaje, que concierne a los aprendizajes que esperamos que logren los alumnos, de acuerdo con la cronología de los objetivos que establece el currículo para cada uno de los niveles de enseñanza, a la precisión de estos aprendizajes y a su riqueza. La siguiente categoría es la limitación de los aprendizajes, que consiste en detectar los errores y/o obstáculos que pueden tener los alumnos con el tópico determinado, generalmente estas dificultades pueden ser sólo ideas o hipótesis de posibles errores, empíricas o conocidas de acuerdo con la experiencia en aula con los alumnos, o errores que han sido documentados por estudios a priori que se han detectado en la práctica, y para los cuales pueden existir propuestas para su tratamiento. La tercera de las categorías son las demandas cognitivas, refiriéndose a las tareas que se proponen para que los alumnos logren los objetivos propuestos en el currículo específico, de tal manera 
Figura 1. Ciclo del Análisis Didáctico (Rico y Fernández-Cano, 2013: 9)

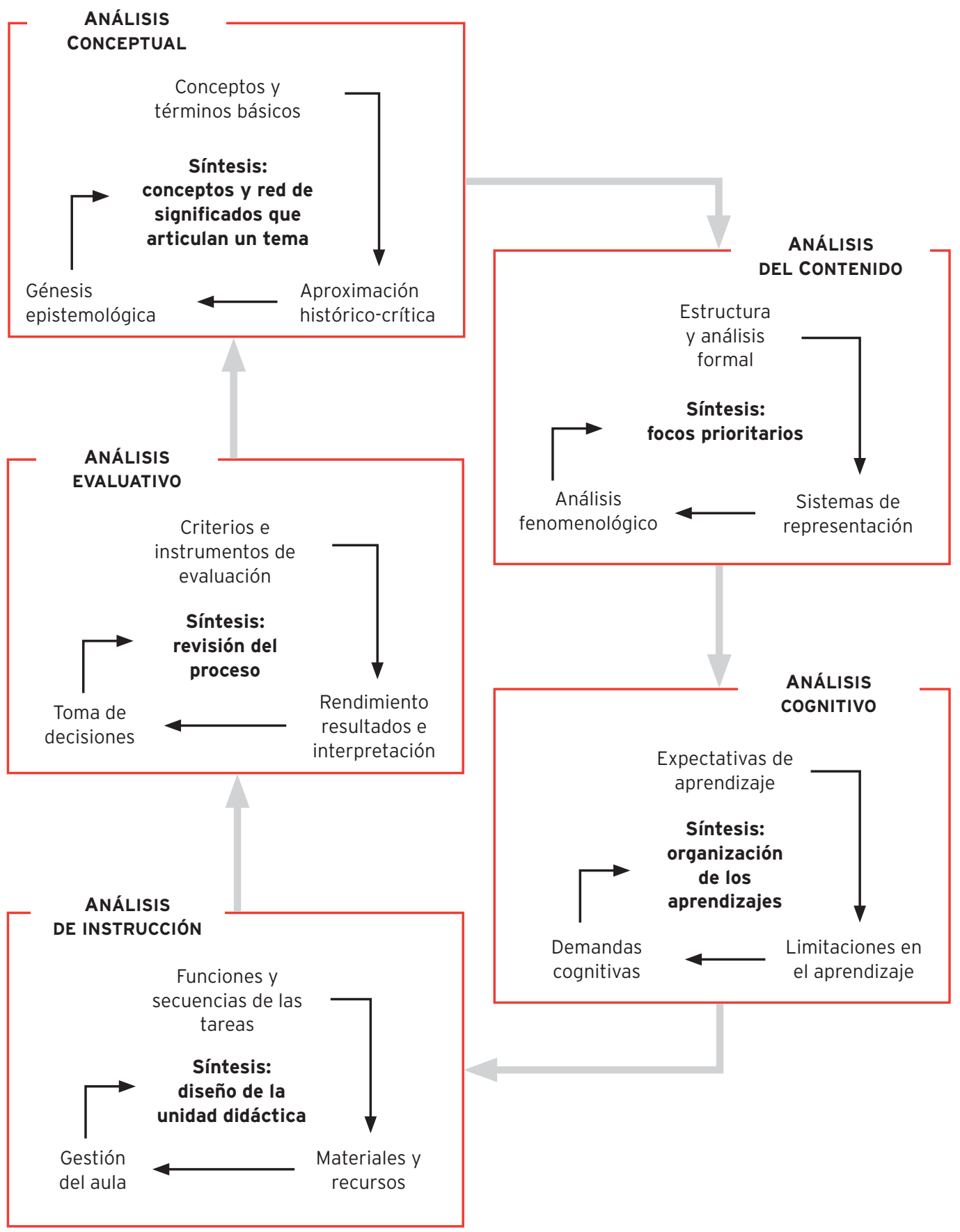


de plantear desafios que se adecuen a las necesidades cognitivas de nuestros alumnos y que les permitan superar los errores relativos a los tópicos en estudio (Rico y Fernández-Cano, 2013).

El análisis de instrucción, que consiste en la transformación y adaptación de los datos relevantes obtenidos en los dos análisis anteriores, responde a las cuestiones de "cómo y cuándo se lleva a cabo la implementación". Esta planificación se puede dar tanto para una clase determinada, para el diseño de un texto o para la planificación general de la coordinación de un centro educativo. Las categorías del análisis de instrucción son tres, la primera de ellas corresponde a las funciones y secuencias de las tareas, consiste en el plan de trabajo para el alumno de acuerdo con una secuencia didáctica específica. La segunda categoría corresponde a los materiales y recursos que serán utilizados en la enseñanza de las matemáticas. La última categoría consiste en la gestión de aula, la organización de la sala de clase, la estructura y gestión de los momentos de la clase (Rico y Fernández-Cano, 2013).

La última etapa del análisis didáctico es el análisis evaluativo, responde a la pregunta “ ¿cuáles han sido los resultados?", lo que involucra tanto la actividad del profesor como la del estudiante. Se compone de tres categorías, la primera, criterios e instrumentos de evaluación, para diagnosticar orientar y evaluar los aprendizajes de los alumnos. Luego tenemos la categoría de rendimiento, resultados e interpretación, de los aprendizajes logrados y no logrados por los alumnos. Finalmente, la tercera categoría corresponde a la toma de decisiones, que evalúa los procesos de enseñanza y aprendizaje diseñados en este ciclo de análisis didáctico, para mejorar y/o modificar las acciones seguidas y comenzar un nuevo ciclo (Rico y Fernández-Cano, 2013).

El trabajo de planificación de unidades didácticas que realizan los futuros profesores, corresponde al nivel de "planificación docente", en donde se toman decisiones respecto al contenido, objetivos de aprendizaje, metodologías de enseñanza y evaluación. Cada uno de los análisis se constituye por algunos organizadores del currículo, herramientas conceptuales y metodológicas, que permiten analizar temas específicos de las matemáticas escolares, usando la información obtenida en el diseño, implementación y evaluación de las unidades (Rico, 1997a).

Se espera que el uso del desde una visión funcional en la formación inicial de profesores (Gómez y González, 2008), permita que los estudiantes desarrollen competencias de diseño, implementación y evaluación de unidades didácticas y la profundización en el conocimiento didáctico y disciplinar que se obtiene luego de realizar idealmente este procedimiento.

\section{Metodología}

Este estudio se enmarca en el paradigma cualitativo, de tipo exploratorio descriptivo (Hernández, Fernández y Baptista, 2010), ya que pretendemos disponer de información que muestre de qué manera es útil para futuros maestros el análisis didáctico. Tomando como instrumentos de recogida de datos las producciones de los estudiantes, realizamos un análisis de contenido de las mismas (Krippendorff, 1990), fijando como unidades referenciales los conjuntos de párrafos que tienen conexión o idea en común.

La formación docente en Chile se realiza en universidades estatales y privadas y en centros de formación técnica. En general, la formación para la enseñanza primaria tiene una duración de entre ocho y diez semestres, dependiendo de si cuenta o no con una mención disciplinaria. La formación universitaria tiene un ingreso por prueba de selección universitaria a nivel nacional, en que postulan a las carreras de acuerdo con el puntaje logrado en dicha evaluación. Existe el apoyo económico con becas del gobierno y becas de las mismas instituciones. El tipo de estudiantes que ingresa a la formación de maestros es diverso, no hay selección por nivel socioeconómico, se discrimina según puntaje obtenido en la Prueba de Selección Universitaria (PSU). 
El contexto de este estudio se enmarca dentro de un acompañamiento didáctico en el Trabajo Final de Titulación de estudiantes de la carrera de Educación Básica, con mención en Matemática de la Universidad Alberto Hurtado. Esta carrera tiene una duración de 10 semestres y los estudiantes obtienen al término de su formación el grado de licenciatura en educación, el título de profesor general básico y una mención disciplinar (matemática, ciencia, lenguaje o historia).

Los sujetos informantes son un grupo de 16 estudiantes de último año (dos hombres y 14 mujeres), que preparan su Trabajo Final de Titulación en la asignatura "Taller de Titulación", en la que realizan el diseño, implementación y análisis de unidades didácticas que abarcan de 8 a 10 horas pedagógicas, para llevarse a cabo en un curso de enseñanza primaria en donde realizan en paralelo su práctica profesional. El Taller se efectúa durante un semestre, una vez a la semana en un tiempo de tres horas pedagógicas, y en él se reúnen todos los estudiantes con el profesor especialista en didáctica de la matemática.

Los contenidos y objetivos de aprendizaje que trabajaron los estudiantes durante el proceso de este estudio, está determinado por la institución en donde realizan su práctica profesional, respondiendo al plan anual propuesto en el currículo nacional vigente. Por ende, no se ven contenidos disciplinares, ya que éstos son profundizados en los cursos dispuestos para ello en los años anteriores (nueve cursos en total: cuatro cursos disciplinares y cinco cursos de didáctica).

En este taller se introduce a los estudiantes en el $\mathrm{AD}$, de tal manera que vayan aplicando dichos aprendizajes en los diseños de sus unidades didácticas. Para ello se organizan los tiempos y los temas tratados en el taller, de acuerdo con la tabla 1.

Cada una de estas tareas fue supervisada por académicos de la especialidad en didáctica de la matemática (Magíster en Didáctica de las Matemáticas de la UGR, misma universidad donde se origina el AD), quienes acompañaron el trabajo de cada uno de los estudiantes.
La relación entre los estudiantes y el académico a cargo, es una relación de por lo menos tres años, ya que el mismo académico dicta tres de los cinco cursos de Didáctica de las Matemáticas de la carrera. En el Taller la comunicación fue de forma personal y en entrevistas individuales entre el futuro profesor y el académico, las cuales se programaron con anticipación, para ver los temas individuales y hacer retro alimentaciones sobre sus avances.

$\mathrm{El}$ académico a cargo de este taller es el investigador principal de este estudio.

El objetivo general del Taller de Titulación es: "Proporcionar la herramienta Análisis Didáctico, como un procedimiento que le permita al futuro profesor diseñar, implementar y evaluar unidades didácticas, desarrollando las competencias necesarias y el conocimiento didáctico que le permita desenvolverse de manera ideal en su labor docente, en un proceso formativo en su último año de carrera profesional docente".

Paralelo al proceso de construcción de su unidad didáctica en el Taller de Titulación, los estudiantes en formación asisten regularmente a sus primeras experiencias laborales, que consiste en estar 15 horas en aula, a cargo de un profesor guía (que trabaja en el colegio y está a cargo de la asignatura de matemática). Durante esta experiencia laboral, el estudiante tiene diversas labores, entre ellas, acompañar al profesor guía en sus clases, asistirlo en el trabajo directo con los alumnos del colegio, apoyar en la revisión de tareas, actividades y evaluaciones que tienen los alumnos, sustentar algunas clases, las cuales son observadas por el tutor didacta de la Universidad.

Finalizar su Trabajo de Titulación, implica el diseño del escrito general de la experiencia y la defensa de su trabajo frente a una comisión evaluadora, en la que hay tres formadores universitarios: un formador en matemática, un formador en didáctica de la matemática y un formador generalista. Esta instancia permite aprobar o reprobar a los estudiantes, lo que permite o no su titulación como profesor. 
Tabla 1. Resumen de las actividades realizadas por los estudiantes durante el Taller

\begin{tabular}{|c|c|c|}
\hline Tarea & Descripción & $\begin{array}{l}\text { Tiempo } \\
\text { destinado }\end{array}$ \\
\hline Diagnóstico institucional & $\begin{array}{l}\text { Investigar las características del colegio, Proyecto Educativo y metodología de } \\
\text { enseñanza. }\end{array}$ & 2 semanas \\
\hline Diagnóstico pedagógico & $\begin{array}{l}\text { Investigar las características de los estudiantes del curso en dónde se realizará } \\
\text { la implementación de la unidad didáctica. Conocer edad, contexto, estilos de } \\
\text { aprendizaje y formas de trabajo. }\end{array}$ & 2 semanas \\
\hline Marco teórico general & $\begin{array}{l}\text { Investigar teorías y metodologías de enseñanza y aprendizaje acordes al } \\
\text { diagnóstico realizado anteriormente. }\end{array}$ & 4 semanas \\
\hline Marco teórico específico & $\begin{array}{l}\text { Primera parte del Análisis Didáctico: } \\
1^{\circ} \text { Análisis conceptual } \\
2^{\circ} \text { Análisis de contenido } \\
3^{\circ} \text { Análisis cognitivo }\end{array}$ & 6 semanas \\
\hline Planificación & $\begin{array}{l}\text { Segunda parte del Análisis Didáctico: } \\
4^{\circ} \text { Análisis de instrucción, diseño de actividades para los estudiantes, de acuerdo } \\
\text { con lo establecido en el currículo nacional. }\end{array}$ & 4 semanas \\
\hline Reflexión & $\begin{array}{l}\text { Última parte del Análisis Didáctico: } \\
5^{\circ} \text { Análisis de actuación }\end{array}$ & 2 semanas \\
\hline
\end{tabular}

Para el análisis de datos nos centraremos primeramente en el análisis conceptual y de contenido, donde el profesor (o futuro profesor) identifica, selecciona y organiza los significados y procedimientos matemáticos relevantes para su unidad didáctica (Lupiáñez, 2009). En concreto consideramos los siguientes elementos:

- Estructura conceptual (del análisis conceptual): establece todas las relaciones y conexiones entre conceptos y procedimientos correspondientes al foco de trabajo en la unidad didáctica

- Sistemas de representación (del análisis de contenido): diferentes formas de representar el contenido y sus relaciones con otros conceptos y procedimientos.
- Fenomenología (del análisis de contenido): considera los fenómenos (contextos, situaciones y problemas) que dan sentido al contenido correspondiente a la unidad didáctica.

Además, miraremos el análisis de instrucción, observando la coherencia entre las tareas diseñadas y el objetivo de clase que se presenta en una planificación diseñada por una futura maestra. También observaremos que el diseño de las tareas esté fundamentado en los análisis previos: conceptual y de contenido, con el fin de evidenciar si la metodología del análisis didáctico es o no una herramienta útil en el proceso de planificación.

Finalmente consideramos algunas reflexiones, las cuales fueron expuestas en este documento, 
correspondientes a la etapa del análisis de actuación y del análisis didáctico.

\section{Análisis y resultados}

$\mathrm{Al}$ analizar las producciones de los alumnos se tienen diversos resultados, profundizaremos en el análisis conceptual, de contenido y el de instrucción.

\section{Análisis conceptual y de contenido}

Los estudiantes deben diseñar un esquema conceptual sobre el contenido a tratar en la unidad didáctica, tomando como referencia los conceptos investigados en el análisis conceptual, el cual realizan en un periodo aproximado de seis semanas, desde que comienzan a indagar los conceptos hasta la entrega del primer informe. Para ello realizan estudios sobre la didáctica del tema o contenido a tratar, extraen información de investigaciones, artículos, conferencias, tesis de magíster, tesis doctorales y textos de la disciplina matemática. El objetivo del esquema conceptual en esta tarea es que puedan organizar, de manera jerárquica, los contenidos y conceptos claves que se ponen en juego durante la unidad didáctica. La figura 2 muestra un ejemplo de este esquema diseñado por uno de los grupos de estudiantes.

Se observa que el estudiante intenta dar a conocer todos los conceptos, elementos y relaciones que se deben poner en juego en el desarrollo de la unidad didáctica que le corresponde diseñar. En esta red conceptual se observan los conceptos previos y aquellos que se verán más adelante sin hacer una discriminación jerárquica sobre ellos. Se observan conceptos de las representaciones y sus relaciones con determinados recursos. Este esquema conceptual le permite estructurar su aprendizaje, para organizar los conceptos previamente o realizar un diagnóstico de ellos (Ontoria, 2003). Sin embargo, no es posible ver una jerarquización coherente a la presentada por el programa de estudio, de acuerdo con el nivel en que debe ser implementado. Lo que sí es observable es que el futuro profesor identifica que existen conceptos y relaciones básicas entre los contenidos a trabajar, evidenciando un avance en su conocimiento como docente. En este caso, el profesor en formación manifiesta que reconoce cierta progresión de los contenidos a enseñar, con los puede trabajar en profundidad.

Otro de los trabajos que debían realizar los estudiantes es diseñar un esquema de representaciones. Un ejemplo de ellos se ilustra en la figura 3, que ilustra las diversas representaciones del concepto matemático decimales, diseñado por una maestra en formación. La estudiante muestra cuatro formas de representar los decimales: simbólica, verbal, manipulativa y gráfica. En cada uno de los aspectos de clasificación nombra una serie de elementos con los que se pueden trabajar los decimales, los que son utilizados en su diseño didáctico de acuerdo con los objetivos de aprendizaje correspondientes a su unidad.

Se observa la dificultad que tiene la estudiante en formación por apreciar representaciones simbólicas en geometría, como serían las representaciones algebraicas de rectas de la forma " $a x+b y+c=0$ ", o las simples sustituciones por letras, como la recta "L", o la recta AP. Sin embargo, es capaz de presentar una clasificación coherente de las distintas representaciones. En este caso, vemos una clasificación entre simbólico, verbal, manipulativo y gráfico. De acuerdo con varios autores, como Font (2000) y Duval (2006), la diversidad de representaciones y la transitividad entre una y otra, permiten que los alumnos tengan una mayor comprensión de los conceptos matemáticos, los mismos examinados anteriormente en el análisis conceptual. Rico (1997a) plantea además, que las representaciones nos ayudan a modelar los fenómenos de estudio. En este sentido, el profesor en formación, acoge esta información entregada en las sesiones teóricas del Taller de Titulación y la evidencia en su trabajo y su aprendizaje.

Por último, dentro del análisis de contenido, los estudiantes realizan un análisis fenomenológico, de los cuales tomamos un ejemplo, analizaremos una 
Figura 2. Esquema conceptual sobre números decimales

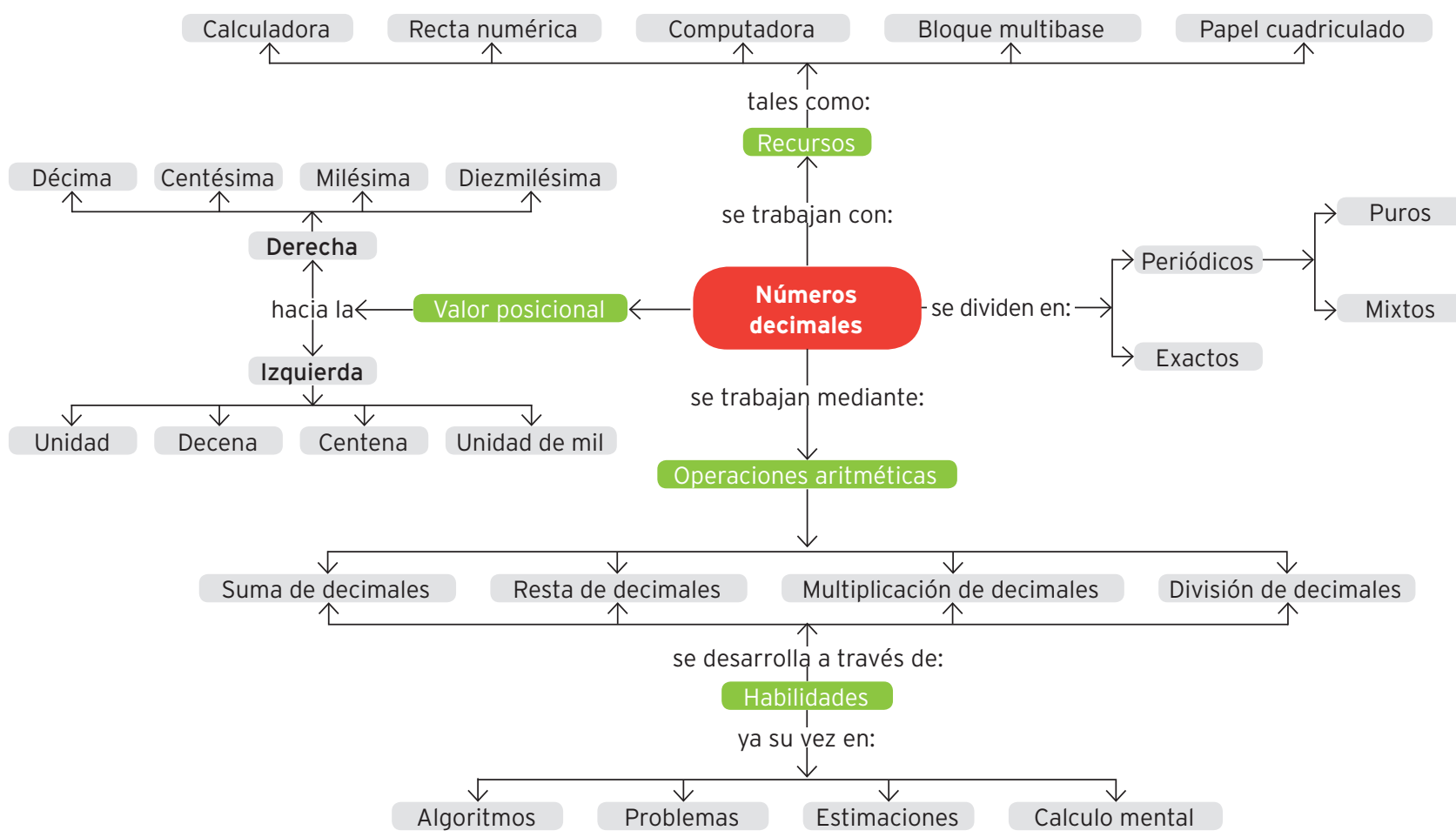

Figura 3. Esquema de representaciones sobre números decimales

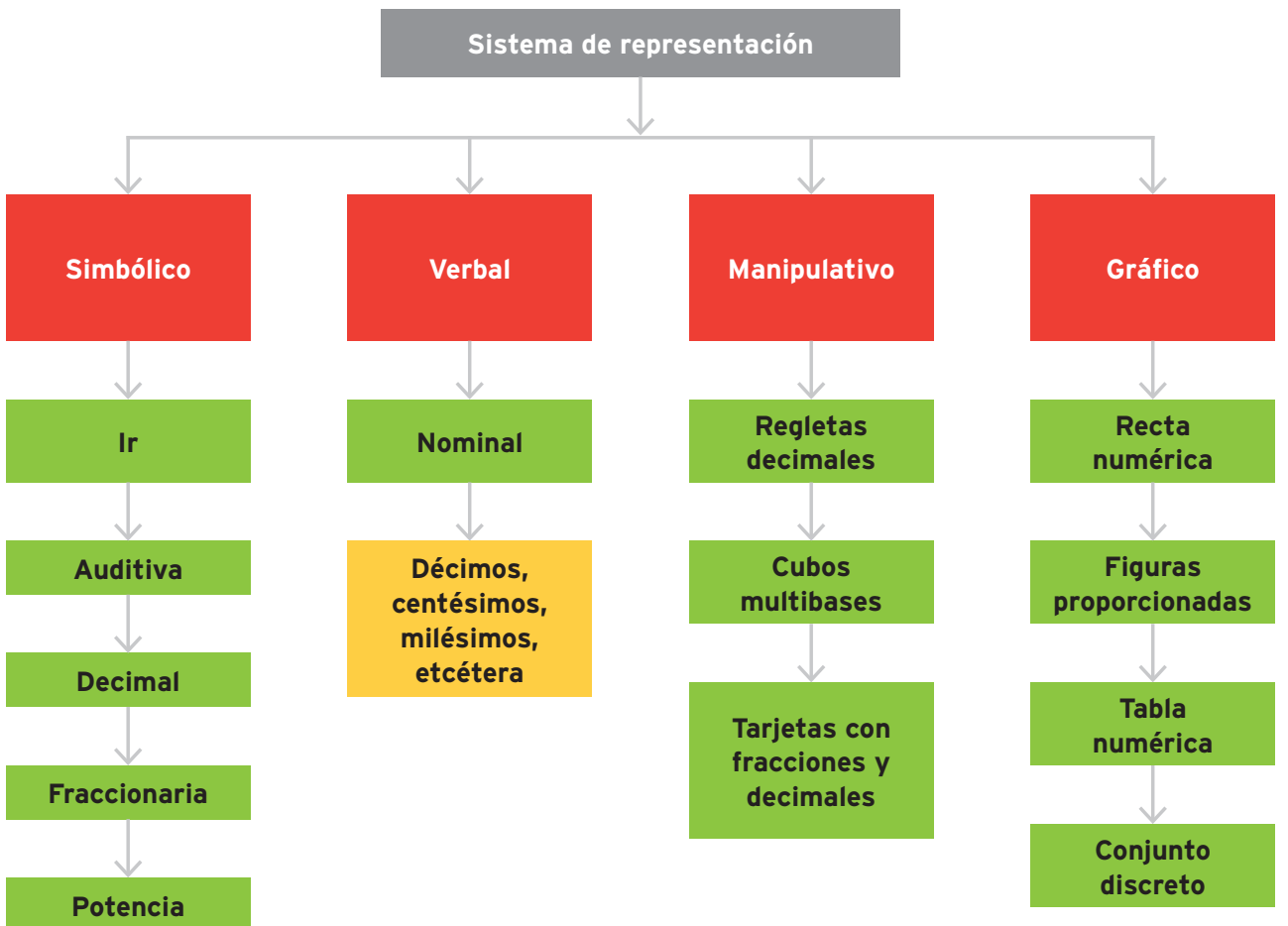


de las producciones de un grupo de estudiantes que se enfocó en el contenido de decimales (figura 4).

La figura 3 muestra una tabla de doble entrada sobre los distintos fenómenos y contextos en los cuales es posible trabajar con los decimales. Este grupo de alumnos realiza una relación entre cada contexto y cada fenómeno, estableciendo así un espacio muestral de distintas situaciones que surgen y en las cuales es posible contextualizar los decimales.

Sin embargo, hay presencia de tres relaciones, en las cuales no es posible identificar una situación de trabajo con decimales, lo que deja abiertas las especulaciones respecto a la investigación a priori que se realizó.

Presentamos la tabla 2 del mismo grupo de trabajo que contiene dos párrafos textuales del informe que realizaron estos estudiantes en formación.

Los estudiantes en formación ponen de manifiesto el profundo análisis fenomenológico que llevaron a cabo, para construir un cuadro síntesis. Además, establecen conexiones entre las situaciones matemáticas y los contextos en los cuales se puede trabajar el contenido correspondiente a su unidad.

Figura 4. Ejemplo de esquema fenomenológico

\begin{tabular}{|c|c|c|c|c|}
\hline & Personales & Educativas o laborales & Públicas & Científicas \\
\hline Medida & $\begin{array}{l}\text { - Manualidades } \\
\text { - Trabajos prácticos } \\
\text { en el hogar } \\
\text { - Bebidas }\end{array}$ & - Construcción & - Carreteras & $\begin{array}{l}\text { - Mediciones } \\
\text { experimentales }\end{array}$ \\
\hline División entera & - Comida & $\begin{array}{l}\text { - Reparación de } \\
\text { campos para la } \\
\text { siembra }\end{array}$ & $\begin{array}{l}\text { - PIB } \\
\text { - Becas } \\
\text { - Subsidio }\end{array}$ & \\
\hline Aproximaciones & $\begin{array}{l}\text { - Memoria de } \\
\text { computadores }\end{array}$ & - Comercio & - Censo & $\begin{array}{l}\text { - Distancias } \\
\text { atronómicas }\end{array}$ \\
\hline Porcentaje & - Comercio & -Impuestos & $\begin{array}{l}\text { - Estadísticas } \\
\text { - Distribución del } \\
\text { ingreso nacional }\end{array}$ & $\begin{array}{l}\text { - Presentación de } \\
\text { resultados }\end{array}$ \\
\hline $\begin{array}{l}\text { Computadores y } \\
\text { calculador }\end{array}$ & - Calculadora & - Excel & $\begin{array}{l}\text { - Software } \\
\text { especializado }\end{array}$ & $\begin{array}{l}\text { - Software } \\
\text { especializado }\end{array}$ \\
\hline Notación científica & & - Ciencias naturales & & $\begin{array}{l}\text { - Mediciones } \\
\text { microscópicas y } \\
\text { macroscópicas }\end{array}$ \\
\hline
\end{tabular}

Tabla 2. Ejemplo de unidades de análisis referidas al análisis fenomenológico realizado por uno de los grupos de estudiantes

Unidades de análisis

Para llevar a cabo el análisis fenomenológico hemos decidido plantear un cuadro de dos ejes en el cual proponemos ejemplos de posibles usos de los decimales junto con definir los espacios de su uso. Para esto hemos trabajado con dos autores diferentes

Las columnas se refieren al criterio clasificador de las tareas matemáticas (OCDE, 2003). Éstas se dividen en situaciones personales, educativas o laborales, públicas y científicas. Éstas se refieren a posibles esferas de aplicación de cualquier concepto matemático en la vida. Por otra parte, los usos prácticos de los decimales están ubicados en las filas. Éstos son: medida, división entera, aproximaciones, porcentaje, computadores y calculadora y notación científica (Castro, 2001). 


\section{Análisis de instrucción}

Este análisis lo abordaremos a través de las producciones de una de las futuras profesoras, quien se plantea una clase sobre figuras 3D. Los futuros maestros debieron revisar los objetivos de aprendizaje e indicadores de los programas de estudio que proporciona el Ministerio de Educación para cada nivel de enseñanza. La futura profesora selecciona del currículo escolar el objetivo para la clase "Identificar aristas, vértices y caras en figuras o dibujos 3D" y la divide en tres partes: inicio, desarrollo y cierre. Realizaremos el análisis según estos tres momentos.

\section{Análisis de tareas en el inicio de la clase}

El inicio lo plantean con la instrucción que se muestra en la tabla 3.

En la primera parte de la tarea (fila 1, de la tabla 1) se puede identificar el propósito de motivar a los alumnos con una tarea novedosa. No se explicita la instrucción para los estudiantes. El objetivo de la tarea tiene poca coherencia con el objetivo de la clase, ya que el propósito de la tarea pretende que los estudiantes identifiquen una figura 3D y no pide que identifiquen las vistas de la figura 3D, como lo dice el objetivo.

El propósito de la segunda parte (fila 2, tabla 1) consiste en identificar el nombre de ciertas partes de una figura 3D, las cuales serán llamadas vértices, aristas y caras, es coherente con el objetivo de la clase. Sin embargo, hay dos aspectos que no son considerados. En primera instancia no considera los posibles errores y dificultades que pudieran tener los alumnos; y en segundo lugar la formulación de la tarea “CCómo llamaremos a ciertas partes?", no utiliza un vocabulario matemático adecuado y está en futuro, es decir, adelantándose a la institucionalización o formalización de los conceptos a trabajar en el desarrollo.

Finalmente se pretende que los estudiantes identifiquen ciertos errores que surgieron durante el desarrollo de la tarea anterior, que es coherente al objetivo antes nombrado.

Tabla 3. Tarea del estereograma

\begin{tabular}{|c|c|c|}
\hline Tarea & Contenidos & Propósito \\
\hline $\begin{array}{l}\text { "Se les muestra a los estudiantes un estereograma, y se } \\
\text { explica cómo deben observarlo" }\end{array}$ & Figuras 3D & Identificar figuras 3D \\
\hline ¿Cómo llamaremos a ciertas partes? & $\begin{array}{l}\text { Elementos básicos de figuras } \\
\text { 3D: aristas, vértices y caras. }\end{array}$ & $\begin{array}{l}\text { Identificar aristas, caras y } \\
\text { vértices en figuras 3D. }\end{array}$ \\
\hline $\begin{array}{l}\text { "Comunican sus resultados, se focalizan posibles errores y } \\
\text { se aclaran" }\end{array}$ & $\begin{array}{l}\text { Elementos básicos de figuras } \\
\text { 3D: aristas, vértices y caras }\end{array}$ & $\begin{array}{l}\text { Identificar posibles errores en } \\
\text { los conceptos. } \\
\text { Sintetizar los aprendizajes }\end{array}$ \\
\hline
\end{tabular}




\section{Análisis de tareas en el desarrollo de la clase}

Para este momento de la clase la futura maestra propone la tabla 4 . El propósito de la tarea es explícito, pues se pretende que el alumno analice y represente figuras 3D y luego comparta sus respuestas. En cambio, el objetivo de la clase es sólo del nivel cognitivo de identificar, surgiendo así la incoherencia entre las habilidades del objetivo de la clase y el propósito de la tarea en función de las habilidades que se ponen en juego.

Los ejemplos de tareas que se presentan tienen relación con las instrucciones de la misma tabla, en donde se puede apreciar que no existe coherencia entre las tareas solicitadas y el objetivo de clase; más aún, no hay coherencia entre la formulación de la tarea y lo que el alumno debe hacer realmente.

\section{Análisis de tareas en el cierre de la clase}

En este último momento de la clase, la futura docente presenta la tabla 5. La tarea es coherente al momento de cierre, a pesar que no se explicitan los conceptos matemáticos a sintetizar.

Tabla 4. Características ocultas de un cuerpo misterioso

\begin{tabular}{|c|c|c|c|c|}
\hline \multicolumn{3}{|c|}{ Tarea } & Contenidos & Propósito \\
\hline \multicolumn{3}{|c|}{$\begin{array}{l}\text { Lee y analiza las características que se presentan a } \\
\text { continuación, dibuja y con ayuda de material concreto } \\
\text { (plasticina y bombillas) intenta recrear el cuerpo 3D oculto. } \\
\text { Finalmente compáralo con el de otros compañeros. }\end{array}$} & Características de figuras 3D & $\begin{array}{l}\text { Analizar las características de } \\
\text { figuras 3D. } \\
\text { Representar figuras 3D, de } \\
\text { manera pictórica y/o concreta. } \\
\text { Compartir con el compañero. }\end{array}$ \\
\hline \multicolumn{3}{|l|}{ Ejemplo 1} & \multirow{3}{*}{$\begin{array}{l}\text { Ejemplo } 1 \\
\text { Figuras 3D } \\
\text { Elementos básicos de figuras } \\
\text { 3D. }\end{array}$} & \multirow{3}{*}{$\begin{array}{l}\text { Ejemplo } 1 \\
\text { Identificar nombre de la figura } \\
\text { 3D, según características } \\
\text { básicas. } \\
\text { Visualizar un cuerpo 3D, a } \\
\text { partir de sus características y } \\
\text { la forma de sus caras. } \\
\text { Representar en 2D, figuras 3D. } \\
\text { Representar con material } \\
\text { concreto figuras 3D. }\end{array}$} \\
\hline Característica & $\begin{array}{l}\text { Nombre } \\
\text { del } \\
\text { cuerpo }\end{array}$ & Dibujo & & \\
\hline $\begin{array}{l}\text { No de caras: } 6 \\
\text { Figura de las caras: } \\
\text { cuadrados } \\
N^{\circ} \text { de Vértices: } 8 \\
N^{\circ} \text { de aristas: } 12\end{array}$ & & & & \\
\hline \multicolumn{3}{|l|}{ Ejemplo 2} & \multirow{3}{*}{$\begin{array}{l}\text { Ejemplo } 2 \\
\text { Figuras 3D. } \\
\text { Elementos básicos de figuras } \\
\text { 3D. }\end{array}$} & \multirow{3}{*}{$\begin{array}{l}\text { Ejemplo } 2 \\
\text { Identificar número de aristas, } \\
\text { vértices y caras. } \\
\text { Identificar nombre de la figura } \\
\text { 3D. } \\
\text { Identificar forma }\end{array}$} \\
\hline Característica & $\begin{array}{l}\text { Nombre } \\
\text { del } \\
\text { cuerpo }\end{array}$ & Dibujo & & \\
\hline $\begin{array}{l}\text { No de caras: } \\
\text { Figura de las caras: } \\
\text { No de Vértices: } \\
\text { No de aristas: }\end{array}$ & & & & \\
\hline
\end{tabular}


Tabla 5. Reflexión final

\begin{tabular}{|l|l|}
\hline \multicolumn{1}{|c|}{ Tarea } & Propósito \\
\hline $\begin{array}{l}\text { Comentar los errores cometidos, reflexionar en } \\
\text { torno a ellos. }\end{array}$ & Reflexionar en torno a los errores cometidos. \\
$\begin{array}{l}\text { Sintetizar los contenidos de la clase brevemente y } \\
\text { registrarlos. }\end{array}$ & Sintetizar. \\
\end{tabular}

\section{Análisis de actuación (reflexiones sobre el uso del análisis didáctico)}

A partir del trabajo realizado los estudiantes llegan a ciertas reflexiones. A continuación mostramos extractos (tabla 6) de conclusiones realizadas por los estudiantes en formación, las cuales evidencian parte de los aprendizajes que obtuvieron, luego de realizar el análisis didáctico. Se muestran dos ejemplos concretos sobre las conclusiones realizadas por los estudiantes.

En el ejemplo 1, los estudiantes concluyen sobre la importancia de realizar este análisis, para reconocer y estructurar los conceptos correspondientes a su unidad didáctica. En el ejemplo 2, los estudiantes identifican los aprendizajes que lograron del concepto analizado, en su caso los números decimales; además son capaces de relacionar el análisis de contenido como sustento para las posteriores etapas del análisis didáctico.

De acuerdo con las reflexiones entregadas por los estudiantes en sus bitácoras de aprendizaje, se pueden observar diversas ideas. El análisis didáctico es un aporte significativo en la formación inicial docente, ya que existe una secuencia lógica de investigación, que les permite a los estudiantes construir conocimientos didácticos: teóricos, técnicos y prácticos. En este sentido, se evidencia una formación didáctica y disciplinar que permite construir conocimiento a los profesores en formación y sustentar el diseño de sus unidades didácticas.

\section{Tabla 6. Algunas conclusiones de los estudiantes sobre el análisis didáctico realizado}

\section{Unidades de análisis}

\section{Ejemplo 1}

Tomando en cuenta los postulados del texto "Capítulo 2. El análisis didáctico: La planificación del aprendizaje desde una perspectiva curricular", reconocemos la importancia de comenzar nuestro análisis didáctico a partir de un análisis de contenido que, como docentes, nos permite reconocer los principales conceptos que están incluidos en nuestro objetivo de aprendizaje. Aplicar esta metodología de trabajo nos permitió ordenar información, realizar un esquema, reconocer las formas de representación y además situar los contenidos en las diversas situaciones y contextos que pueden ser utilizados.

\section{Ejemplo 2}

A través de los diversos análisis que hemos realizado, hemos aprendido bastante acerca de los números decimales en los tres niveles en los que trabajamos. Esto nos provee de herramientas para enfrentar el análisis cognitivo y las posteriores etapas del diseño, implementación y evaluación de nuestra unidad didáctica. Además es una herramienta que nos puede servir el día de mañana para planificar y ejecutar nuestras propias unidades y clases en el ámbito laboral. 


\section{Conclusiones}

De acuerdo con los datos proporcionados en nuestra investigación, se puede evidenciar que el análisis didáctico está presente en el desarrollo de su implementación, ya que los estudiantes hacen una investigación a priori de los contenidos disciplinares y didácticos considerados en su unidad didáctica, de tal manera de hacerse cargo de los aprendizajes de sus alumnos, los cuales son plasmados en los informes finales de su trabajo de titulación.

También se puede observar que existe un intento por comprender en profundidad los conceptos y temas que se ponen en juego durante el análisis didáctico, ya que hay evidencias de ello. En primer lugar, se cuenta con mapas conceptuales, que si bien no tienen una jerarquización coherente, sí hay presencia de los conceptos pedagógicos y disciplinares a considerar en la construcción de su unidad didáctica. Esto les permite tener una visión más amplia sobre la progresión de los conceptos a trabajar en sus clases, por ejemplo, desde donde comienzan y hasta dónde pueden avanzar, así como responden a cuestiones del $\mathrm{AD}$ como ¿qué es el conocimiento matemático?, ya que el análisis conceptual les permite profundizar en los conceptos matemáticos desde la estructura matemática.

En segundo lugar, se constata que los estudiantes para profesor profundizan sobre las distintas representaciones de los conceptos que se ponen en juego durante la unidad didáctica, lo que les permite diseñar de manera diversa las tareas de aprendizaje para sus alumnos, permitiendo una mayor comprensión de los conceptos matemáticos (Duval, 2006; Font, 2000).

Por último, se evidencia una profundización de los futuros profesores para analizar los diferentes contextos y situaciones en los cuales se pueden trabajar los conceptos matemáticos a planificar, de esta forma el profesor en formación podrá diseñar o escoger tareas de aprendizajes diversas, de acuerdo con contextos, situaciones o fenómenos más significativos.
Estos elementos nos dan muestra de la utilidad que ha tenido para los futuros profesores el desarrollo del análisis didáctico, y acogiéndolo e interpretándolo como una herramienta provechosa para el diseño, implementación y análisis de unidades didácticas, objetivo de nuestro estudio. De acuerdo con lo anterior y con nuestro objetivo general en este estudio, es posible evidenciar la presencia de estos análisis, los que permiten a nuestros profesores en formación tomar conciencia de la complejidad del trabajo de planificación e implementación de unidades didácticas y las relaciones existentes entre los aspectos disciplinares y didácticos de los contenidos, lo cual se pone de manifiesto en sus reflexiones finales.

Por otro lado, del análisis de instrucción, se observa que él y la futura docente se plantean su clase en términos de inicio, desarrollo y cierre. Estos tres momentos parece que les dieran seguridad a la hora de planificar una clase, seguridad que puede estar basada en la mantención de una estructura organizada. A pesar de ello, los tres momentos no llevan coherencia con el objetivo de la clase, más aún el objetivo recién es abordado en el momento de cierre de la clase. Esto nos deja ver que futuros maestros tiene carencia de sentido respecto al planteamiento del objetivo de la clase versus la formulación de la misma.

Se destaca además en este estudio, la toma de conciencia de nuestros estudiantes sobre su propio aprendizaje, sobre sus fortalezas y debilidades en cuanto a sus competencias de diseño, implementación y evaluación de unidades didácticas, ya que si bien es cierto que en su primer acercamiento con el análisis didáctico lograron profundizar en los aspectos conceptuales, fenomenológicos y sobre las representaciones de los conceptos claves de sus unidades, sin embargo, hay una brecha entre este conocimiento y su manifestación en las tareas de clase, como también en aspectos metodológicos claves como la construcción de esquemas conceptuales.

En cuanto al aspecto teórico del conocimiento didáctico, los futuros maestros analizan cuestiones 
como: qué contenidos encierran los temas de la matemática, cómo se conectan los contenidos aprendidos en cursos anteriores, los contenidos a enseñar y los contenidos futuros, entre otros, dando respuestas a cuestiones del análisis didáctico como: ¿Qué es la enseñanza? ¿Para qué sirve el conocimiento?

En otros trabajos nuestros (Valenzuela, 2012) resaltamos que es fundamental observar la historia, la fenomenología y otros organizadores del currículo, para el diseño, planificación y evaluación de unidades didácticas. Sostenemos que los organizadores del currículo son parte esencial al iniciar los cursos de formación docente en matemática, así como su uso secuencial en la enseñanza de las matemáticas, lo que debe estar presente en toda la formación inicial docente, no sólo en el último año, para que los cambios y aplicaciones directas del AD se fortalezca en su último año de estudios y sobre todo en su práctica profesional.

Nos planteamos pues la relevancia de contar con herramientas que permitan al futuro profesor mirar con sentido sus planificaciones, de forma que pueda de profundizar en el objetivo propuesto por ellos. Dejamos abiertas muchas ventanas, que nos pueden dar luz hacia las respuestas de cómo mejorar la práctica de futuros maestros al alero de sus planificaciones de clases.

De acuerdo con esta experiencia, se puede constatar que es necesario diseñar cursos de formación inicial docente en matemáticas, que profundicen sobre el significado matemático y didáctico del contenido. Son múltiples las investigaciones que han trabajado en lo que debe saber un profesor de cualquier nivel educativo y área de aprendizaje. A lo largo de los años han ido cambiando los enfoques, antes se resaltaba el conocimiento matemático, para ir evolucionando hacia aspectos centrados en la didáctica general (Rojas, Flores y Carrillo, 2013), Shulman (1986) profundiza en el proceso de aprendizaje que los estudiantes de pedagogía en general construyen, identificando conocimientos pedagógicos específicos de una disciplina.

A partir de estas ideas, nos preguntamos: ¿Qué conocimiento debería poseer un profesor de matemática para ejercer su profesión y cómo llega a establecerlo? ¿Cómo va adquiriendo el profesor en formación inicial el conocimiento para llegar a ser un profesor de matemáticas? Son muchos los autores que han centrado sus investigaciones para dar respuestas a estas interrogantes, planteando diferentes modelos de conocimiento, que arrancan de la idea inicial de Shulman (1986). En el ámbito de la didáctica de las matemáticas destacamos las líneas abiertas por el grupo de investigación en la Universidad de Michigan, Ball, Thames y Phelps (2008); en la Universidad de Huelva, Sosa y Carrillo (2010), y el planteado por Rowland, Huckstep y Thwaites (2005) con su "Knowledge Quartet" (KQ), entre otras líneas. De acuerdo con estos avances respecto al conocimiento didáctico y disciplinar que debiera tener un profesor de matemáticas, el uso del análisis didáctico se ha manifestado como una herramienta útil para el futuro docente de matemática chileno, que aporta para desarrollar las competencias necesarias para analizar los conceptos matemáticos desde aspectos didácticos y disciplinares, lo que se pone de manifiesto en el diseño, implementación y evaluación de unidades didácticas por los futuros profesores. 


\section{Referencias}

Aros, Daniela y Nicole Astudillo (2015), Resignificación de las propiedades de intersección, unión y complemento de un evento, Valparaíso, Pontificia Universidad Católica de Valparaíso.

Ávalos, Beatriz (2002), Profesores para Chile, historia de un proyecto, Santiago, Ministerio de Educación.

Ball, Deborah, Mark Thames y Geoffrey Phelps (2008), "Content knowledge for teaching: What makes it special?", en Fournal of Teacher Education, vol. 59, núm. 5, pp. 389-407.

Blömeke, Sigrid, Ute Suhl y Gabriele Kaiser (2011), "Teacher education effectiveness: quality and equity of future primary teachers' mathematics and mathematics pedagogical content knowledge", en Fournal of Teacher Education, vol. 62, núm. 2, pp. 154171.

Castro, Enrique(2001), "Números decimales", en Didáctica de la Matemática en la educación primaria, núm. 1, pp. 315345.

Cox, Cristian (2007), "Educación en el Bicentenario: dos agendas y calidad en las políticas", en Pensamiento Educativo, vol. 40, núm. 1, pp. 175-204.

Doyle, Walter (1933), "Academic work", en Review of Educational Research, vol. 53, núm. 2, pp. 159-199.

Duval, Raymond(2006), "Un tema crucial en la educación matemática: la habilidad para cambiar el registro de representación", en La Gaceta de la Real Sociedad Matemática Española, vol. 9, núm.1, pp. 143-168.

Flores, Pablo, Pedro Gómez y Antonio Marín (2013), Apuntes sobre análisis de instrucción, módulo 4 de MAD, Documento no publicado, Bogotá, Universidad de los Andes, <http://funes.uniandes.edu.co/2061/>.

Flores, Pablo y Luis Rico (2015), Enseñanza y aprendizaje de las matemáticas en Educación Primaria, Madrid, Ediciones Pirámide.

Fonseca. J. y J. Reyes (2015), La dispersión de los datos en las medidas de tendencia central, Valparaíso, Pontificia Universidad Católica de Valparaíso.

Font, Vicent (2000), "Algunos puntos de vista sobre las representaciones en didáctica de las matemáticas”, en Philosophy of Mathematics Education Fournal, vol. 14, pp. $1-35$.

Gómez, Pedro(2007),Desarrollo del conocimiento didáctico en un plan de formación inicial de profesores de matemáticas de secundaria, Granada, Departamento de Didáctica de la Matemática de la Universidad de Granada, <http:// funes.uniandes.edu.co/444/>. [Fecha de consulta:...].

Gómez, Pedro y María José González (2008), "Mathematics knowledge for teaching within a functional perspective of preservice teacher training", en ICME 11 Topic Study Group 27, Monterrey, <http:// funes.uniandes.edu.co/418/>.

Gómez, Pedro y María José González (2013), "Diseño de planes de formación de profesores de matemáticas basados en el análisis didáctico”, en Luis Rico, José Luis Lupiáñez y Marta Molina (eds.), Análisis didáctico en educación matemática: metodología de investigación, formación de profesores e innovación curricular, Granada, Comares, pp. 121-140.

Hernández, Roberto, Carlos Fernández y Pilar Baptista (2010), Metodología de la investigación, México, McGrawHill.

Krippendorff, Klaus (1990), Metodología de análisis de contenido: teoría y práctica, Barcelona, Paidós.

León, Gloria (2011), Unidad didáctica: fracciones, Granada, Universidad de Granada.

Lupiáñez, José Luis (2009), Expectativas de aprendizaje y planificación curricular en un programa de formación inicial de profesores de matemáticas de secundaria, Granada, Universidad de Granada, <http://tiny u rl. com/8k4wt9j>.

Lupiáñez, José Luis y Luis Rico (2008), "Análisis didáctico y formación inicial de profesores: competencias y capacidades en el aprendizaje de los escolares", en PNA, vol. 3, núm. 1, pp. 35-48.

Lupiáñez,José Luis y Luis Rico (2006), “Análisis didáctico y formación inicial de profesores: organización de competencias y capacidades de los escolares en el caso 
de los números decimales", en INDIVISA, Madrid, Centro Superior de Estudios Universitarios La Salle, vol. IV, 47-58.

Ministerio de Educación (MINEDUC) (2012), Evaluación INICIA, <http://www.evaluacioninicia.cl/ed01.html>. Organización para la Cooperación y el Desarrollo Económicos (OCDE) (2003), The pisa 2003 Assessment framework. Mathematics, Reading, science and problema, solving, knowledge and skills, París, OCDE.

Ontoria, Antonio (2003), Aprender con Mapas Conceptuales, Madrid, Ediciones Narcea.

Ortega, Manuel (2012), Unidad didáctica. Sucesiones matemáticas. Progresiones aritméticas y geométricas, Granda, Universidad de Granada.

Ortiz, Valeria (2013), Unidad didáctica: celebremos nuestra patria: geometria, Santiago, Universidad Alberto Hurtado.

Ortúzar, Soledad, Carolina Flores, Carolina Milesi y Cristian Cox (2009), Aspectos de la formación inicial docente y su influencia en el rendimiento académico de los alumnos. Camino al Bicentenario, propuestas para Chile, Santiago, PUC-Concurso de Políticas Públicas.

Pedraja-Rejas, Liliana, Carmen Araneda, Emilio Rodríguez-Ponce y Juan Rodríguez-Ponce (2012), "Calidad en la formación inicial docente: evidencia empírica en las universidades chilenas", en Formación universitaria, vol. 5, núm. 4, pp. 15-26.

Ponte,Joaoda(2005), "Gestãocurricularem Matemática", en GTI (ed.), O professor e o desenvolvimento curricular, Lisboa, APM, pp. 11-34.

Rico, Luis (1997a), Bases teóricas del currículo de matemáticas de educación secundaria, Madrid, Síntesis.

Rico, Luis (1997b), "Los organizadores del currículo de matemáticas”, en Luis Rico (ed.), La educación matemática en la enseñanza secundaria, Barcelona, Horsori, pp. 3959.

Rico, Luis (2007), "La competencia matemática en PISA", en Pensamiento numérico y algebraico, vol. 1, núm. 2, pp. 47-66.

Rico, Luis e Isidoro Segovia (2001), "Unidades didácticas: organizadores", en Enrique Castro (coord.), Didáctica de la matemática en la educación primaria, Madrid, Síntesis, pp. 83-104.

Rico, Luis y Antonio Fernández-Cano (2013), "Análisis didáctico y metodología de investigación”, en Luis Rico, José Luis Lupiáñez y Marta Molina (eds.), Análisis didáctico en Educación Matemática: metodología de investigación, formación de profesores e innovación curricular, Granada, Comares, pp. 1-22.

Rico, Luis, José Luis Lupiáñez y Marta Molina (2013), Análisis didáctico en educación matemática, Granada, Comares.

Rojas, Nielka (2014), “Caracterización del conocimiento especializado del profesor de matemáticas: un estudio de casos", tesis doctoral, Granada, Universidad de Granada.

Rojas, Nielka, Pablo Flores y José Carrillo (2013), "Caracterización del conocimiento matemático para la enseñanza de los números racionales", en Avances de investigación en Educación Matemática, núm. 4, pp. 47-64.

Rowland, Tim, Peter Huckstep y Anne Thwaites (2005), "Elementary teachers' mathematics subject knowledge: The knowledge quartet and the case of Naomi”, en Fournal of Mathematics Teacher Education, vol. 8, núm. 3, pp. 255-281.

Ruiz, Francisca (2013), Unidad didáctica: de punta y codo a regla y compás, construir en geometría desde una experiencia premilitar, Santiago, Universidad Alberto Hurtado.

Ruiz, Natalia (2009), "Medios y recursos parala enseñanza de la geometría en la educación obligatoria”, en Revista Electrónica de Didácticas Específicas, núm. 3, pp. 13-27.

Segovia Isidoro y Luis Rico (2011), Matemáticas para Maestros de Educación Primaria, Madrid, Pirámide.

Shulman, Lee (1986), "Those who understand: knowledge growth in teaching”, en Educational Researcher, vol. 15, núm. 2, pp. 4-14.

Sosa, Landy y José Carrillo (2010), "Caracterización del conocimiento matemático para la enseñanza (MKT) de matrices en bachillerato", en Investigación en Educación Matemática XIV, pp. 569-580.

Valenzuela, M. (2012), "Uso de materiales manipulativos 
didácticos en la enseñanza de la geometría. Un estudio sobre algunos colegios en Chile", Trabajo fin de máster, Universidad de Granada, España.
Villar, Luis (1986), Pensamiento de los profesores y toma de decisiones, Sevilla, Servicio de Publicaciones de la Universidad.

Cómo citar este artículo:

Valenzuela-Molina, Macarena, Elisabeth Ramos-Rodríguez, Lylian-Iris González-Plate y JocelynLysette Portugal-Villar (2018), "El análisis didáctico como base de un curso en la formación inicial de profesores de educación primaria", en Revista Iberoamericana de Educación Superior (RIES), México, UNAM-IISUE/Universia, vol. Ix, núm. 25, pp. 118-137, http://dx.doi.org/10.22201/ iisue.20072872e.2019.25.345 [consulta: fecha de última consulta]. 